\title{
Qualitative Behavior of Solutions of Tenth-Order Recursive Sequence Equation
}

\author{
E. M. Elsayed $\left(\mathbb{D},{ }^{1,2}\right.$ B. S. Alofi $\left(\mathbb{D},{ }^{1} \text { and Abdul Qadeer Khan }{ }^{3}\right)^{3}$ \\ ${ }^{1}$ King Abdulaziz University, Faculty of Science, Mathematics Department, P.O. Box 80203, Jeddah 21589, Saudi Arabia \\ ${ }^{2}$ Department of Mathematics, Faculty of Science, Mansoura University, Mansoura, Egypt \\ ${ }^{3}$ Department of Mathematics, University of Azad Jammu and Kashmir, Muzaffarabad 13100, Pakistan
}

Correspondence should be addressed to Abdul Qadeer Khan; abdulqadeerkhan1@gmail.com

Received 17 September 2021; Revised 14 November 2021; Accepted 31 December 2021; Published 14 February 2022

Academic Editor: Baogui Xin

Copyright (c) 2022 E. M. Elsayed et al. This is an open access article distributed under the Creative Commons Attribution License, which permits unrestricted use, distribution, and reproduction in any medium, provided the original work is properly cited.

Most nonlinear difference equations have exact solutions that are not always possible to obtain theoretically. As a result, a large number of researchers investigate several qualitative aspects of difference equations in order to predict their lengthy behavior. The goal of our research is to obtain the solutions of a tenth-order difference equation $U_{n+1}=U_{n-9} U_{n-5} U_{n-1} / U_{n-7} U_{n-3}( \pm$ $\left.1 \pm U_{n-9} U_{n-5} U_{n-1}\right), n \geq 0$, where the initial values are positive real numbers. Stability and periodicity are also investigated.

\section{Introduction}

Solving the difference equation is one of the problems that is difficult to determine the solvability. The aim of this study is to solve difference equation of the tenth order and solve four specific cases of the following difference equation:

$$
U_{n+1}=\frac{U_{n-9} U_{n-5} U_{n-1}}{U_{n-7} U_{n-3}\left( \pm 1 \pm U_{n-9} U_{n-5} U_{n-1}\right)}, \quad n \geq 0,
$$

where the initial conditions $U_{-9}, U_{-8}, U_{-7}, U_{-6}, U_{-5}, U_{-4}, U_{-3}$, $U_{-2}, U_{-1}, U_{0}$ are the arbitrary positive real numbers. We also provide some properties of solutions such as periodicity in two cases and stability in the other two cases. Difference equations are used in a variety of probability problems such as hypergeometric, binomial, and poison distribution. Differential equations are related to difference equations in the same way that discrete mathematics and continuous mathematician are related. Difference equations are of importance to computer scientists for a variety of reasons. For example, when estimating the cost of an algorithm in big-O notation, converting a difficult differential problem to a nearly equivalent difference equation is the first step in solving. The study of asymptotic stability of nonlinear rational difference equations of high order is a difficult but rewarding task. It is particularly beneficial for analyzing the characteristics of mathematical models using different applications such as biological systems. The main topic in study is that the difference equations theory has been the asymptotic behavior of rational form of difference equation.

In addition, various nonlinear trends in science and engineering can be modeled by this type of equation, and the solution of this type of equation provides a prototype for the development of theory [1]. In the literature, many applications theories' differences equations have been investigated. El-Dessoky [2] investigated the behavior properties of the solutions of the rational difference equation:

$$
U_{n+1}=a U_{n}+\frac{b U_{n} U_{n-3}}{c U_{n-4}+d U_{n-3}} .
$$

Ghazela et al. [3] researched the analytic qualities of sixth-order difference equations: 


$$
U_{n+1}=\frac{b U_{n-5}}{c U_{n-2} U_{n-5}+d} .
$$

Al-Matrafi and Al-Zubaidi [4] achieved global and local stability and forms of positive periodic solutions for two types of recursive equations:

$$
U_{n+1}=a U_{n-1} \pm \frac{b U_{n-1} U_{n-4}}{c U_{n-4}-d U_{n-6}}
$$

Exploring some properties of the behavior of solutions appropriate to the class of recursive equation:

$$
U_{n+1}=a U_{n-1} \pm \frac{b U_{n-1} U_{n-3}}{c U_{n-3}-d U_{n-5}}
$$

was the prime objective for Alayachi et al. in [5]. Sadiq and Kalim [6] studied solutions, equilibrium points, and periodicity of four types of difference equations:

$$
U_{n+1}=\frac{U_{n-20}}{ \pm 1 \pm U_{n-6} U_{n-13} U_{n-20}} .
$$

Elsayed in [7] was able to get the solutions to this difference questions:

$$
U_{n+1}=\frac{U_{n} U_{n-2} U_{n-4}}{U_{n-1} U_{n-3}\left( \pm 1 \pm U_{n} U_{n-2} U_{n-4}\right)} .
$$

For more articles in this direction, we refer the reader to [8-14] and references cited therein.

\section{The First Case:}

$$
\begin{aligned}
& U_{n+1}==U_{n-9} U_{n-5} U_{n-1} / U_{n-7} U_{n-3}\left(1+U_{n-9} U_{n-5}\right. \\
& \left.U_{n-1}\right)
\end{aligned}
$$

The aim of this section is studying the solutions form of the particular case:

$$
U_{n+1}=\frac{U_{n-9} U_{n-5} U_{n-1}}{U_{n-7} U_{n-3}\left(1+U_{n-9} U_{n-5} U_{n-1}\right)}, \quad n=0,1, \ldots
$$

Theorem 1. Assume that $\left(U_{n}\right)_{n=-9}^{\infty}$ are solutions of difference equations. Then, for $n=0,1,2, \ldots$, we see that all solutions of equation (8) are given by the following formulas:

$$
\begin{gathered}
U_{12 n-9}=\frac{A \prod_{k=0}^{n-1}(1+6 k A E I)}{\prod_{k=0}^{n-1}(1+(6 k+2) A E I)}, \\
U_{12 n-3}=\frac{G \prod_{k=0}^{n-1}(1+(6 k+3) A E I)}{\prod_{k=0}^{n-1}(1+(6 k+5) A E I)},
\end{gathered}
$$

$$
\begin{aligned}
& U_{12 n-8}=\frac{B \prod_{k=0}^{n-1}(1+6 k B F J)}{\prod_{k=0}^{n-1}(1+(6 k+2) B F J)}, \\
& U_{12 n-2}=\frac{H \prod_{k=0}^{n-1}(1+(6 k+3) B F J)}{\prod_{k=0}^{n-1}(1+(6 k+5) B F J)}, \\
& U_{12 n-7}=\frac{C \prod_{k=0}^{n-1}(1+(6 k+1) A E I)}{\prod_{k=0}^{n-1}(1+(6 k+3) A E I)}, \\
& U_{12 n-1}=\frac{I \prod_{k=0}^{n-1}(1+(6 k+4) A E I)}{\prod_{k=0}^{n-1}(1+(6 k+6) A E I)}, \\
& U_{12 n-6}=\frac{D \prod_{k=0}^{n-1}(1+(6 k+1) B F J)}{\prod_{k=0}^{n-1}(1+(6 k+3) B F J)}, \\
& U_{12 n}=\frac{J \prod_{k=0}^{n-1}(1+(6 k+4) B F J)}{\prod_{k=0}^{n-1}(1+(6 k+6) B F J)}, \\
& U_{12 n-5}=\frac{E \prod_{k=0}^{n-1}(1+(6 k+2) A E I)}{\prod_{k=0}^{n-1}(1+(6 k+4) A E I)}, \\
& U_{12 n+1}=\frac{A E I \prod_{k=0}^{n-1}(1+(6 k+5) A E I)}{C G(1+A E I) \prod_{k=0}^{n-1}(1+(6 k+7) A E I)} \\
& U_{12 n-4}=\frac{F \prod_{k=0}^{n-1}(1+(6 k+2) B F J)}{\prod_{k=0}^{n-1}(1+(6 k+4) B F J)}, \\
& U_{12 n+2}=\frac{B F J \prod_{k=0}^{n-1}(1+(6 k+5) B F J)}{D H(1+B F J) \prod_{k=0}^{n-1}(1+(6 k+7) B F J)},
\end{aligned}
$$

where $U_{-9}=A, \quad U_{-8}=B, U_{-7}=C, U_{-6}=D, U_{-5}=E$, $U_{-4}=F, U_{-3}=G, U_{-2}=H, U_{-1}=I$, and $U_{0}=J$.

Proof. For $n=1$, the result holds. Now suppose that $n>0$ and that our assumption holds for $n-1$, that is,

$$
\begin{aligned}
& U_{12 n-21}=\frac{A \prod_{k=0}^{n-2}(1+6 k A E I)}{\prod_{k=0}^{n-2}(1+(6 k+2) A E I)}, \\
& U_{12 n-15}=\frac{G \prod_{k=0}^{n-2}(1+(6 k+3) A E I)}{\prod_{k=0}^{n-2}(1+(6 k+5) A E I)}, \\
& U_{12 n-20}=\frac{B \prod_{k=0}^{n-2}(1+6 k B F J)}{\prod_{k=0}^{n-2}(1+(6 k+2) B F J)}, \\
& U_{12 n-14}=\frac{H \prod_{k=0}^{n-2}(1+(6 k+3) B F J)}{\prod_{k=0}^{n-2}(1+(6 k+5) B F J)}, \\
& U_{12 n-19}=\frac{C \prod_{k=0}^{n-2}(1+(6 k+1) A E I)}{\prod_{k=0}^{n-2}(1+(6 k+3) A E I)}, \\
& U_{12 n-13}=\frac{I \prod_{k=0}^{n-2}(1+(6 k+4) A E I)}{\prod_{k=0}^{n-2}(1+(6 k+6) A E I)},
\end{aligned}
$$




$$
\begin{aligned}
& U_{12 n-18}=\frac{D \prod_{k=0}^{n-2}(1+(6 k+1) B F J)}{\prod_{k=0}^{n-2}(1+(6 k+3) B F J)}, \\
& U_{12 n-12}=\frac{J \prod_{k=0}^{n-2}(1+(6 k+4) B F J)}{\prod_{k=0}^{n-2}(1+(6 k+6) B F J)}, \\
& U_{12 n-17}=\frac{E \prod_{k=0}^{n-2}(1+(6 k+2) A E I)}{\prod_{k=0}^{n-2}(1+(6 k+4) A E I)}, \\
& U_{12 n-11}=\frac{A E I \prod_{k=0}^{n-2}(1+(6 k+5) A E I)}{C G(1+A E I) \prod_{k=0}^{n-2}(1+(6 k+7) A E I)}, \\
& U_{12 n-16}=\frac{F \prod_{k=0}^{n-2}(1+(6 k+2) B F J)}{\prod_{k=0}^{n-2}(1+(6 k+4) B F J)}, \\
& U_{12 n-10}=\frac{B F J \prod_{k=0}^{n-2}(1+(6 k+5) B F J)}{D H(1+B F J) \prod_{k=0}^{n-2}(1+(6 k+7) B F J)} .
\end{aligned}
$$

Now, we find from equation (8) that

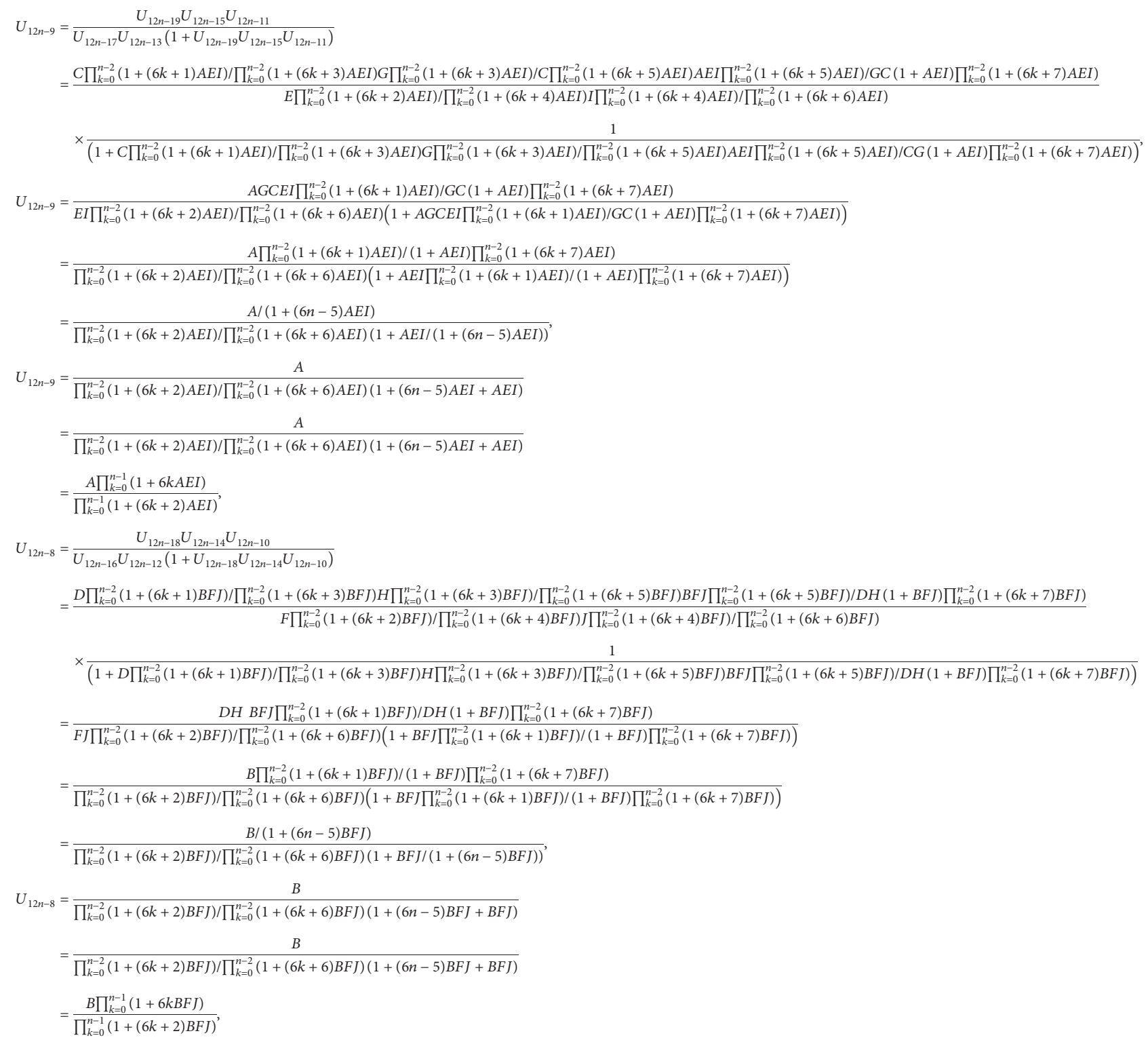




$$
\begin{aligned}
& U_{12 n-7}=\frac{U_{12 n-17} U_{12 n-13} U_{12 n-9}}{U_{12 n-15} U_{12 n-11}\left(1+U_{12 n-17} U_{12 n-13} U_{12 n-9}\right)} \\
& =\frac{E \prod_{k=0}^{n-2}(1+(6 k+2) A E I) / \prod_{k=0}^{n-2}(1+(6 k+4) A E I) I \prod_{k=0}^{n-2}(1+(6 k+4) A E I) / \prod_{k=0}^{n-2}(1+(6 k+6) A E I) A \prod_{k=0}^{n-1}(1+6 k A E I) / \prod_{k=0}^{n-1}(1+(6 k+2) A E I)}{(1+2) A E I)} \\
& G \prod_{k=0}^{n-2}(1+(6 k+3) A E I) / \prod_{k=0}^{n-2}(1+(6 k+5) A E I) A E I \prod_{k=0}^{n-2}(1+(6 k+5) A E I) / C G(1+A E I) \prod_{k=0}^{n-2}(1+(6 k+7) A E I) \\
& \times \frac{1}{\left(1+E \prod_{k=0}^{n-2}(1+(6 k+2) A E I) / \prod_{k=0}^{n-2}(1+(6 k+4) A E I) I \prod_{k=0}^{n-2}(1+(6 k+4) A E I) / \prod_{k=0}^{n-2}(1+(6 k+6) A E I) A \prod_{k=0}^{n-1}(1+6 k A E I) / \prod_{k=0}^{n-1}(1+(6 k+2) A E I)\right)} \\
& \prod_{k=0}^{n-1}(1+6 k A E I) /(1+(6 n-4) A E I) \prod_{k=0}^{n-2}(1+(6 k+6) A E I)
\end{aligned}
$$

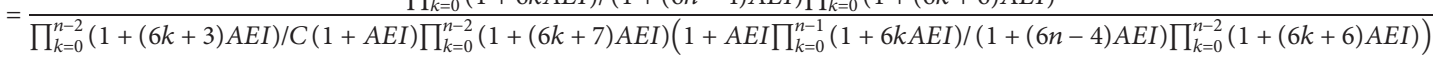

$$
\begin{aligned}
& =\frac{1 /(1+(6 n-4) A E I)}{\prod_{k=0}^{n-2}(1+(6 k+3) A E I) / C(1+A E I) \prod_{k=0}^{n-2}(1+(6 k+7) A E I)(1+A E I /(1+(6 n-4) A E I))} \\
& =\frac{C(1+A E I)}{\prod_{k=0}^{n-2}(1+(6 k+3) A E I) / \prod_{k=0}^{n-2}(1+(6 k+7) A E I)((1+(6 n-4) A E I)+A E I)} \\
& =\frac{C \prod_{k=0}^{n-1}(1+(6 k+1) A E I)}{\prod_{k=0}^{n-1}(1+(6 k+3) A E I)}, \\
& U_{12 n-6}=\frac{U_{12 n-16} U_{12 n-12} U_{12 n-8}}{U_{12 n-14} U_{12 n-10}\left(1+U_{12 n-16} U_{12 n-12} U_{12 n-8}\right)} \\
& =\frac{F \prod_{k=0}^{n-2}(1+(6 k+2) B F J) / \prod_{k=0}^{n-2}(1+(6 k+4) B F J) J \prod_{k=0}^{n-2}(1+(6 k+4) B F J) / \prod_{k=0}^{n-2}(1+(6 k+6) B F J) B \prod_{k=0}^{n-1}(1+6 k B F J) / \prod_{k=0}^{n-1}(1+(6 k+2) B F J)}{H \prod_{k=0}^{n-2}(1+(6 k+3) B F J) / \prod_{k=0}^{n-2}(1+(6 k+5) B F J) B F J \prod_{k=0}^{n-2}(1+(6 k+5) B F J) / D H(1+B F J) \prod_{k=0}^{n-2}(1+(6 k+7) B F J)} \\
& \times 1 /\left(1+\frac{F \prod_{k=0}^{n-2}(1+(6 k+2) B F J)}{\prod_{k=0}^{n-2}(1+(6 k+4) B F J)} \frac{\prod_{k=0}^{n-2}(1+(6 k+4) B F J)}{\prod_{k=0}^{n-2}(1+(6 k+6) B F J)} B \prod_{k=0}^{n-1}(1+6 k B F J) / \prod_{k=0}^{n-1}(1+(6 k+2) B F J)\right) \\
& =\frac{\prod_{k=0}^{n-1}(1+6 k B F J) /(1+(6 n-4) B F J) \prod_{k=0}^{n-2}(1+(6 k+6) B F J)}{\prod_{k=0}^{n-2}(1+(6 k+3) B F J) / D(1+B F J) \prod_{k=0}^{n-2}(1+(6 k+7) B F J)\left(1+B F J \prod_{k=0}^{n-1}(1+6 k B F J) /(1+(6 n-4) B F J) \prod_{k=0}^{n-2}(1+(6 k+6) B F J)\right)} \\
& =\frac{1 /(1+(6 n-4) B F J)}{\prod_{k=0}^{n-2}(1+(6 k+3) B F J) / D(1+B F J) \prod_{k=0}^{n-2}(1+(6 k+7) B F J)(1+B F J /(1+(6 n-4) B F J))} \\
& =\frac{C \prod_{k=0}^{n-1}(1+(6 k+1) B F J)}{\prod_{k=0}^{n-1}(1+(6 k+3) B F J)},
\end{aligned}
$$

Also, we can prove the other relations. The proof is complete.

Theorem 2. Equation (8) has a unique equilibrium point $\bar{U}=0$, which is nonhyperbolic.

Proof. To obtain equilibrium points of (8),

$$
\bar{U}=\frac{\bar{U}^{3}}{\bar{U}^{2}\left(1+\bar{U}^{3}\right)} .
$$

Thus,

$$
\begin{aligned}
\bar{U}^{3}\left(1+\bar{U}^{3}\right) & =\bar{U}^{3}, \\
\bar{U}^{3}\left(1+\bar{U}^{3}-1\right) & =0, \\
\bar{U}^{6} & =0 .
\end{aligned}
$$

Hence, $\bar{U}=0$ is the equilibrium point of equation (8). Define a function $h:(0, \infty)^{5} \longrightarrow(0, \infty)$, such that

$$
h(r, s, t, u, v)=\frac{r s t}{u v(1+r s t)} \text {. }
$$

Then,

$$
\begin{aligned}
& h_{r}(r, s, t, u, v)=\frac{s t}{u v(1+r s t)^{2}}, \\
& h_{s}(r, s, t, u, v)=\frac{r t}{u v(1+r s t)^{2}}, \\
& h_{t}(r, s, t, u, v)=\frac{r s}{u v(1+r s t)^{2}}, \\
& h_{u}(r, s, t, u, v)=-\frac{r s t}{u^{2} v(1+r s t)}, \\
& h_{v}(r, s, t, u, v)=-\frac{r s t}{u v^{2}(1+r s t)} .
\end{aligned}
$$

Therefore,

$$
\begin{aligned}
h_{r}(\bar{U}, \bar{U}, \bar{U}, \bar{U}, \bar{U}) & =1, \\
h_{s}(r, s, t, u, v) & =1, \\
h_{t}(\bar{U}, \bar{U}, \bar{U}, \bar{U}, \bar{U}) & =1, \\
h_{u}(r, s, t, u, v) & =-1, \\
h_{v}(\bar{U}, \bar{U}, \bar{U}, \bar{U}, \bar{U}) & =-1 .
\end{aligned}
$$


It follows the characteristic equation given by

$$
\lambda^{10}-\lambda^{9}+\lambda^{8}-\lambda^{6}+\lambda^{4}-\lambda^{2}=0
$$

Hence,

$$
\lambda^{8}-\lambda^{7}+\lambda^{6}-\lambda^{4}+\lambda^{2}-1=0 .
$$

Clearly, $\lambda=1$ is one root of equation (17). Therefore, the equilibrium point is nonhyperbolic.

We provide numerical examples for equation (8) in order to confirm the results of this section.

Example 1. Assume the initial conditions are $U_{-9}=7$, $U_{-8}=15, U_{-7}=9, U_{-6}=5, U_{-5}=10, U_{-4}=8, U_{-3}=16$, $U_{-2}=10, U_{-1}=6$, and $U_{0}=11$ (Figure 1 ).

Example 2. Suppose that $U_{-9}=0.1, U_{-8}=0.2, U_{-7}=0.3$, $U_{-6}=0.4, \quad U_{-5}=0.5, \quad U_{-4}=0.1, \quad U_{-3}=0.2, \quad U_{-2}=0.3$, $U_{-1}=0.4$, and $U_{0}=0.5$ (Figure 2).

\section{Second Case:}

$$
\begin{aligned}
& U_{n+1}==U_{n-9} U_{n-5} U_{n-1} / U_{n-7} U_{n-3} \\
& \left(1-U_{n-9} U_{n-5} U_{n-1}\right)
\end{aligned}
$$

The solutions to difference equations

$$
U_{n+1}=\frac{U_{n-9} U_{n-5} U_{n-1}}{U_{n-7} U_{n-3}\left(1-U_{n-9} U_{n-5} U_{n-1}\right)}, \quad n=0,1, \ldots,
$$

are investigated in this section.

Theorem 3. Assume that $\left\{U_{n}\right\}_{n=-9}^{\infty}$ are solutions of difference equations. Then, for $n=0,1,2, \ldots$, we see that all solutions of equation (19) are given by the following formulas:

$$
\begin{gathered}
U_{12 n-9}=\frac{A \prod_{k=0}^{n-1}(1-6 k A E I)}{\prod_{k=0}^{n-1}(1-(6 k+2) A E I)}, \\
U_{12 n-3}=\frac{G \prod_{k=0}^{n-1}(1-(6 k+3) A E I)}{\prod_{k=0}^{n-1}(1-(6 k+5) A E I)}, \\
U_{12 n-8}=\frac{B \prod_{k=0}^{n-1}(1-6 k B F J)}{\prod_{k=0}^{n-1}(1-(6 k+2) B F J)}, \\
U_{12 n-2}=\frac{H \prod_{k=0}^{n-1}(1-(6 k+3) B F J)}{\prod_{k=0}^{n-1}(1-(6 k+5) B F J)}, \\
U_{12 n-7}=\frac{C \prod_{k=0}^{n-1}(1-(6 k+1) A E I)}{\prod_{k=0}^{n-1}(1-(6 k+3) A E I)}, \\
U_{12 n-1}=\frac{I \prod_{k=0}^{n-1}(1-(6 k+4) A E I)}{\prod_{k=0}^{n-1}(1-(6 k+6) A E I)}, \\
U_{12 n-6}=\frac{D \prod_{k=0}^{n-1}(1-(6 k+3) B F J)}{\prod_{k=0}^{n-1}(1-(6 k+1) B F J)}, \\
U_{12 n}=\frac{J \prod_{k=0}^{n-1}(1-(6 k+4) B F J)}{\prod_{k=0}^{n-1}(1-(6 k+4) B F J)},
\end{gathered}
$$

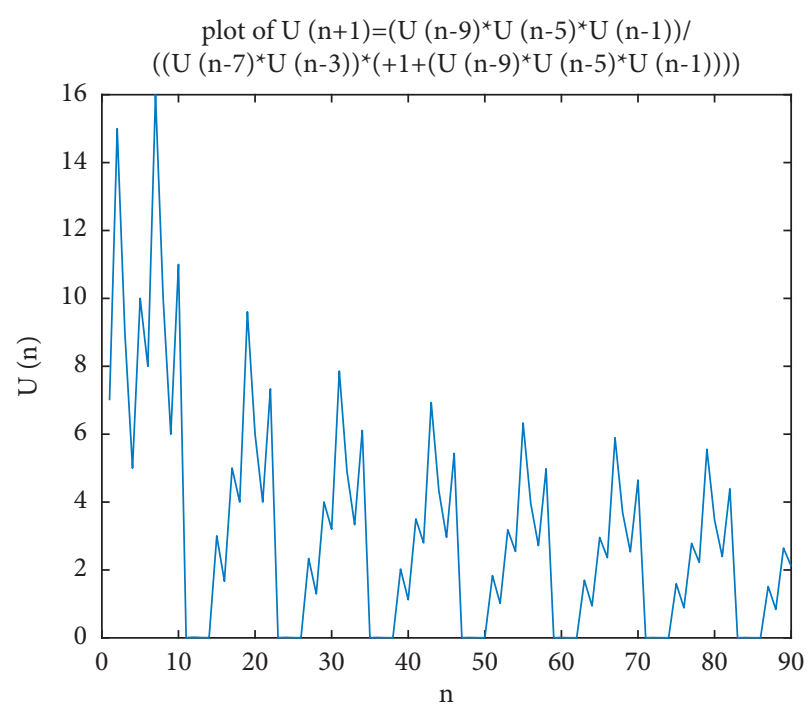

Figure 1: The solution of equation (8) when $U_{-9}=7, U_{-8}=15$, $U_{-7}=9, U_{-6}=5, U_{-5}=10, U_{-4}=8, U_{-3}=16, U_{-2}=10, U_{-1}=6$, and $U_{0}=11$.

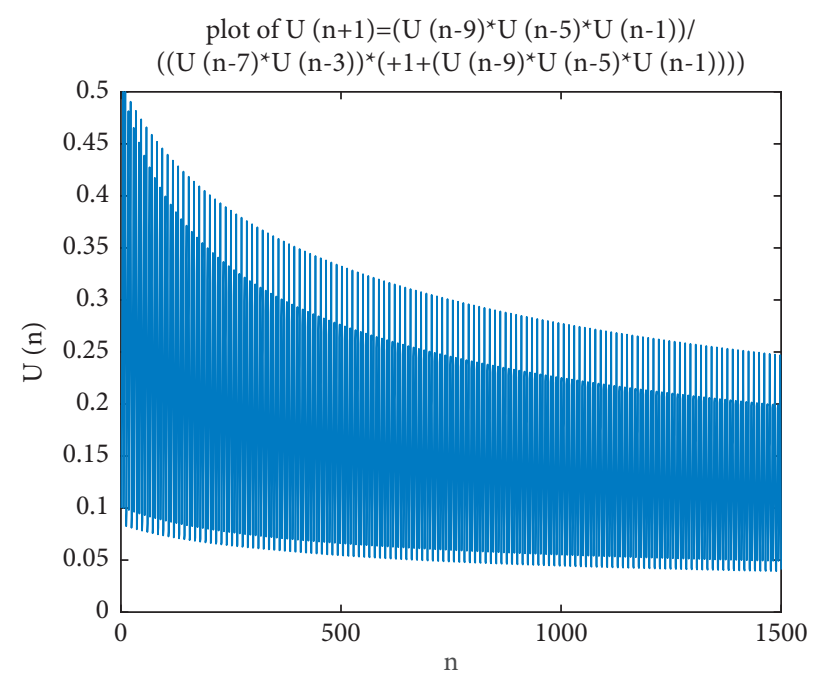

Figure 2: The local stability of equilibrium point of equation (8) when $U_{-9}=0.1, \quad U_{-8}=0.2, \quad U_{-7}=0.3, \quad U_{-6}=0.4, \quad U_{-5}=0.5$, $U_{-4}=0.1, U_{-3}=0.2, U_{-2}=0.3, U_{-1}=0.4$, and $U_{0}=0.5$.

$$
\begin{aligned}
U_{12 n-5} & =\frac{E \prod_{k=0}^{n-1}(1-(6 k+2) A E I)}{\prod_{k=0}^{n-1}(1-(6 k+4) A E I)}, \\
U_{12 n+1} & =\frac{A E I \prod_{k=0}^{n-1}(1-(6 k+5) A E I)}{(1-A E I) \prod_{k=0}^{n-1}(1-(6 k+7) A E I)}, \\
U_{12 n-4} & =\frac{F \prod_{k=0}^{n-1}(1-(6 k+2) B F J)}{\prod_{k=0}^{n-1}(1-(6 k+4) B F J)}, \\
U_{12 n+2} & =\frac{B F J \prod_{k=0}^{n-1}(1-(6 k+5) B F J)}{(1-B F J) \prod_{k=0}^{n-1}(1-(6 k+7) B F J)},
\end{aligned}
$$


where $U_{-9}=A, \quad U_{-8}=B, \quad U_{-7}=C, \quad U_{-6}=D, \quad U_{-5}=E$, $U_{-4}=F, U_{-3}=G, U_{-2}=H, U_{-1}=I$, and $U_{0}=J$.

Proof. The proof is identical to the method to prove Theorem 1.

Theorem 4. Equation (19) has a unique equilibrium point $\bar{U}=0$, which is nonhyperbolic.

Proof. To obtain equilibrium points of equation (19),

$$
\bar{U}=\frac{\bar{U}^{3}}{\bar{U}^{2}\left(1-\bar{U}^{3}\right)} \text {. }
$$

Thus,

$$
\begin{aligned}
\bar{U}^{3}\left(1-\bar{U}^{3}\right) & =\bar{U}^{3}, \\
\bar{U}^{3}\left(1-\bar{U}^{3}-1\right) & =0, \\
\bar{U}^{6} & =0 .
\end{aligned}
$$

Hence, $\bar{U}=0$ is the equilibrium point of equation (19). Define a function $h:(0, \infty)^{5} \longrightarrow(0, \infty)$, such that

$$
h(r, s, t, u, v)=\frac{r s t}{u v(1-r s t)} .
$$

Then,

$$
\begin{aligned}
& h_{r}(r, s, t, u, v)=\frac{s t}{u v(1-r s t)^{2}}, \\
& h_{s}(r, s, t, u, v)=\frac{r t}{u v(1-r s t)^{2}}, \\
& h_{t}(r, s, t, u, v)=\frac{r s}{u v(1-r s t)^{2}}, \\
& h_{u}(r, s, t, u, v)=-\frac{r s t}{u^{2} v(1-r s t)} \\
& h_{v}(r, s, t, u, v)=-\frac{r s t}{u v^{2}(1-r s t)} .
\end{aligned}
$$

Therefore,

$$
\begin{aligned}
h_{r}(\bar{U}, \bar{U}, \bar{U}, \bar{U}, \bar{U}) & =1, \\
h_{s}(r, s, t, u, v) & =1, \\
h_{t}(\bar{U}, \bar{U}, \bar{U}, \bar{U}, \bar{U}) & =1, \\
h_{u}(r, s, t, u, v) & =-1, \\
h_{v}(\bar{U}, \bar{U}, \bar{U}, \bar{U}, \bar{U}) & =-1 .
\end{aligned}
$$

It follows the characteristic equation given by

$$
\lambda^{10}-\lambda^{9}+\lambda^{8}-\lambda^{6}+\lambda^{4}-\lambda^{2}=0 .
$$

Hence,

$$
\lambda^{8}-\lambda^{7}+\lambda^{6}-\lambda^{4}+\lambda^{2}-1=0 .
$$

Clearly, $\lambda=1$ is one root of equation (26). Therefore, the equilibrium point is nonhyperbolic.

Example 3. We consider the present numerical example for equation (19) for confirming the results of this section where the initial conditions are $U_{-9}=4, U_{-8}=12, U_{-7}=6$, $U_{-6}=2, U_{-5}=8, U_{-4}=3, U_{-3}=11, U_{-2}=5, U_{-1}=2$, and $U_{0}=7$ (Figure 3).

Example 4. We provide another numerical example for equation (19) with initial values $U_{-9}=0.1, U_{-8}=0.2$, $U_{-7}=0.3, \quad U_{-6}=0.4, \quad U_{-5}=0.5, \quad U_{-4}=0.1, \quad U_{-3}=0.2$, $U_{-2}=0.3, U_{-1}=0.4$, and $U_{0}=0.5$ (Figure 4$)$.

\section{Third Case:}

$$
\begin{aligned}
& U_{n+1}==U_{n-9} U_{n-5} U_{n-1} / U_{n-7} U_{n-3}\left(-1-U_{n-9}\right. \\
& \left.U_{n-5} U_{n-1}\right)
\end{aligned}
$$

The goal of this section is to obtain the solutions form of the particular case:

$$
U_{n+1}=\frac{U_{n-9} U_{n-5} U_{n-1}}{U_{n-7} U_{n-3}\left(-1-U_{n-9} U_{n-5} U_{n-1}\right)}, \quad n=0,1, \ldots
$$

Theorem 5. Every solution $\left\{U_{n}\right\}_{n=-9}^{\infty}$ of equation (28) is periodic with period twelve, and it is in the form

$\left\{A, B, C, D, E, F, G, H, I, J,-\frac{A E I}{C G(A E I+1)},-\frac{B F J}{D H(B F J+1)}, \ldots\right\}$

or

$$
\begin{aligned}
& U_{12 n-9}=A, \\
& U_{12 n-8}=B, \\
& U_{12 n-7}=C, \\
& U_{12 n-6}=D, \\
& U_{12 n-5}=E, \\
& U_{12 n-4}=F, \\
& U_{12 n-3}=G, \\
& U_{12 n-2}=H, \\
& U_{12 n-1}=I, \\
& U_{12 n}=J,
\end{aligned}
$$

$$
U_{12 n+2}=-\frac{B F J}{D H(B F J+1)},
$$




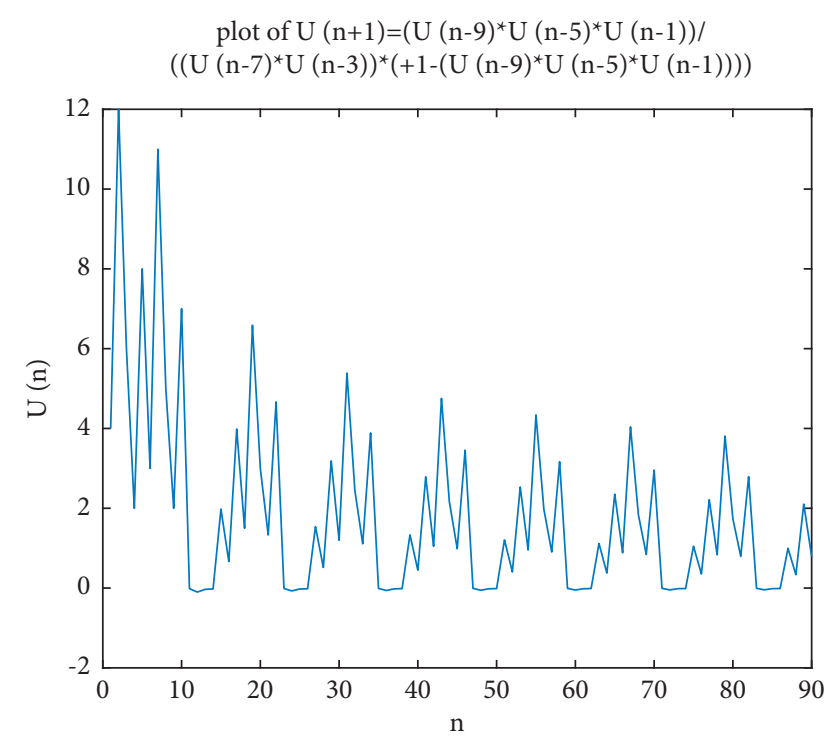

FIGURE 3: The solution of equation (19) when $U_{-9}=4, U_{-8}=12$, $U_{-7}=6, U_{-6}=2, U_{-5}=8, U_{-4}=3, U_{-3}=11, U_{-2}=5, U_{-1}=2$, and $U_{0}=7$.

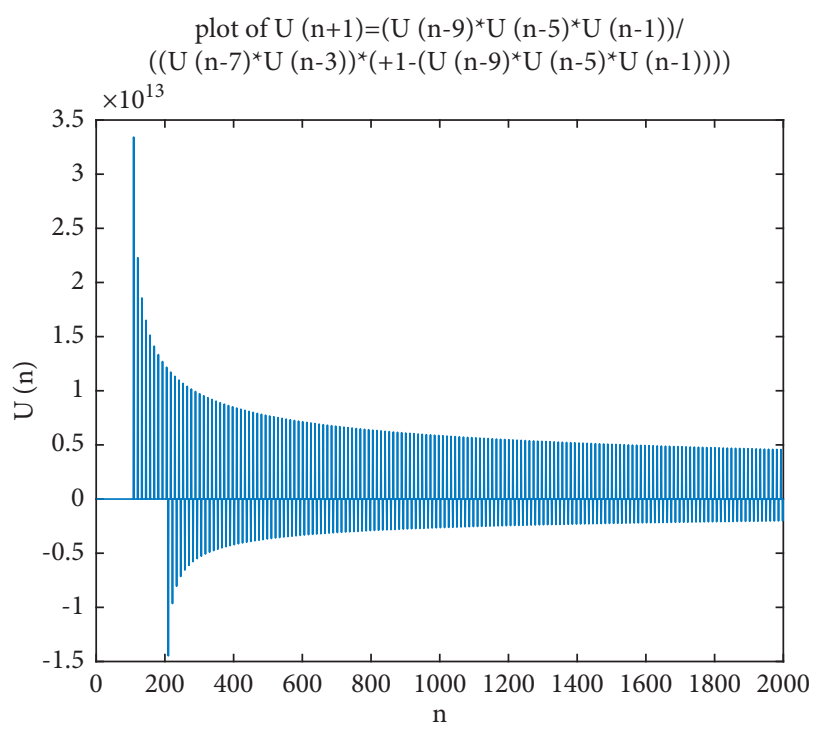

Figure 4: The local stability of equilibrium point of equation (19) with $U_{-9}=0.1, \quad U_{-8}=0.2, \quad U_{-7}=0.3, \quad U_{-6}=0.4, \quad U_{-5}=0.5$, $U_{-4}=0.1, U_{-3}=0.2, U_{-2}=0.3, U_{-1}=0.4$, and $U_{0}=0.5$.

where $U_{-9}=A, \quad U_{-8}=B, \quad U_{-7}=C, \quad U_{-6}=D, U_{-5}=E$, $U_{-4}=F, U_{-3}=G, U_{-2}=H, U_{-1}=I$, and $U_{0}=J$.

Proof. From $n=1$,

$$
\begin{aligned}
& U_{12 n-21}=A, \\
& U_{12 n-20}=B, \\
& U_{12 n-19}=C, \\
& U_{12 n-18}=D,
\end{aligned}
$$

$$
\begin{aligned}
& U_{12 n-17}=E, \\
& U_{12 n-16}=F, \\
& U_{12 n-15}=G, \\
& U_{12 n-14}=H, \\
& U_{12 n-13}=I, \\
& U_{12 n-12}=J, \\
& U_{12 n-11}=-\frac{A E I}{C G(A E I+1)}, \\
& U_{12 n-10}=-\frac{B F J}{D H(B F J+1)} .
\end{aligned}
$$

From equation (28), we see that

$$
\begin{aligned}
U_{12 n-9} & =\frac{U_{12 n-19} U_{12 n-15} U_{12 n-11}}{U_{12 n-17} U_{12 n-13}\left(-1-U_{12 n-19} U_{12 n-15} U_{12 n-11}\right)} \\
& =\frac{C G(-A E I / C G(A E I+1))}{E I(-1-C G(-A E I / C G(A E I+1)))} \\
& =A, \\
U_{12 n-8} & =\frac{U_{12 n-18} U_{12 n-14} U_{12 n-10}}{U_{12 n-16} U_{12 n-12}\left(-1-U_{12 n-18} U_{12 n-14} U_{12 n-10}\right)} \\
& =\frac{D H(-B F J / D H(B F J+1))}{F J(-1-D H(-B F J / D H(B F J+1)))} \\
& =B, \quad
\end{aligned}
$$$$
U_{12 n-7}=\frac{U_{12 n-17} U_{12 n-13} U_{12 n-9}}{U_{12 n-15} U_{12 n-11}\left(-1-U_{12 n-17} U_{12 n-13} U_{12 n-9}\right)}
$$$$
=\frac{A E I}{G(-A E I / C G(A E I+1))(-1-A E I)}
$$$$
=C \text {, }
$$$$
U_{12 n-6}=\frac{U_{12 n-16} U_{12 n-12} U_{12 n-8}}{U_{12 n-14} U_{12 n-10}\left(-1-U_{12 n-16} U_{12 n-12} U_{12 n-8}\right)}
$$$$
=\frac{B F J}{H(-B F J / D H(B F J+1))(-1-B F J)}
$$$$
=D \text {, }
$$

$$
\begin{aligned}
U_{12 n-5} & =\frac{U_{12 n-15} U_{12 n-11} U_{12 n-7}}{U_{12 n-13} U_{12 n-9}\left(-1-U_{12 n-15} U_{12 n-11} U_{12 n-7}\right)} \\
& =\frac{G(-A E I / C G(A E I+1)) C}{I A(-1-G(-A E I / C G(A E I+1)) C)} \\
& =E,
\end{aligned}
$$

$$
\begin{aligned}
U_{12 n-4} & =\frac{U_{12 n-14} U_{12 n-10} U_{12 n-6}}{U_{12 n-12} U_{12 n-8}\left(-1-U_{12 n-14} U_{12 n-10} U_{12 n-6}\right)} \\
& =\frac{H(-B F J / D H(B F J+1)) D}{J B(-1-H(-B F J / D H(B F J+1)) D)} \\
& =F,
\end{aligned}
$$




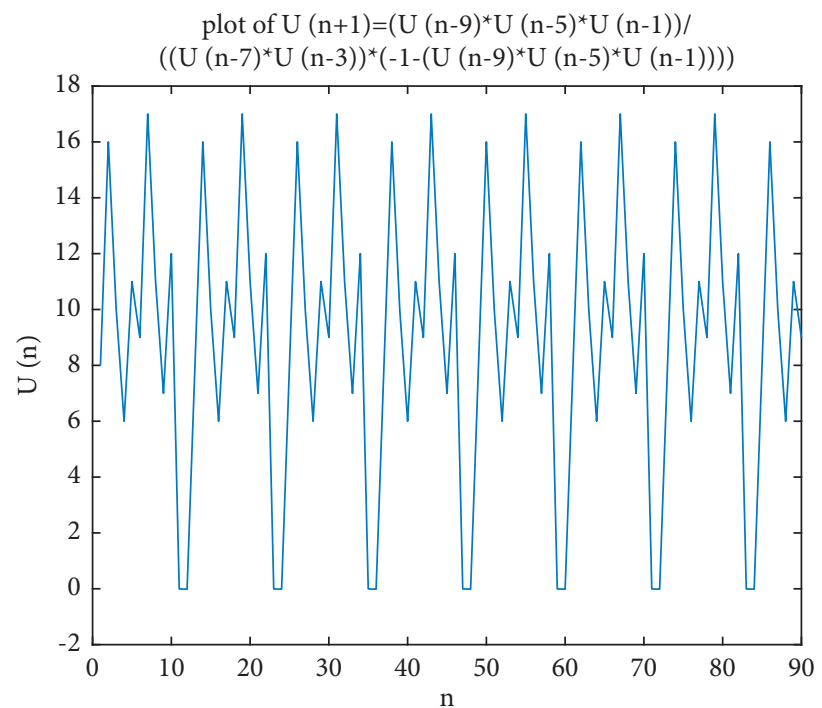

Figure 5: The solution of equation (28) with period twelve when $U_{-9}=8, U_{-8}=16, U_{-7}=10, U_{-6}=6, U_{-5}=11, U_{-4}=9, U_{-3}=17$, $U_{-2}=11, U_{-1}=7$, and $U_{0}=12$.

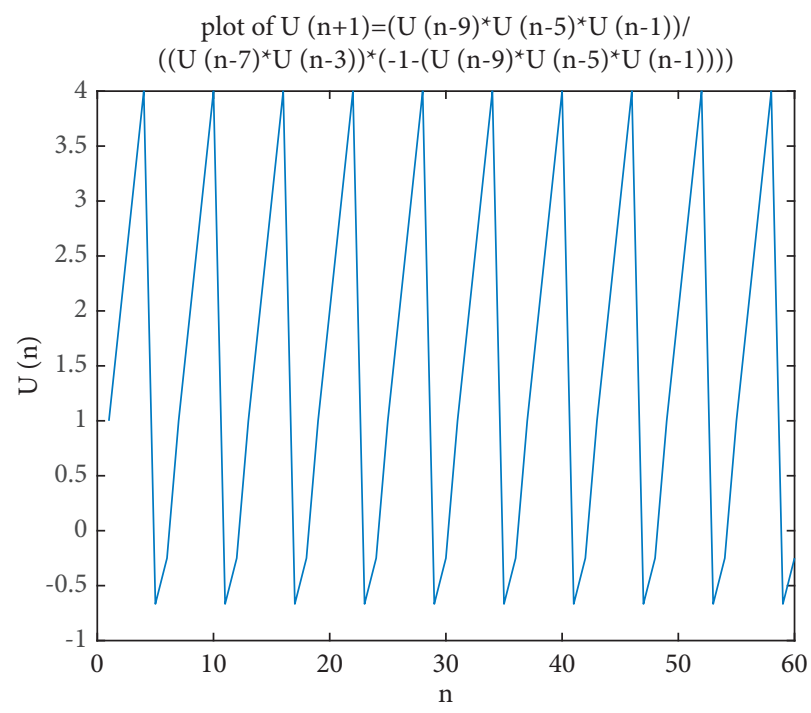

Figure 6: The solution of equation (28) with period six when initial values satisfies the conditions in Theorem 6 , that are $U_{-9}=1, U_{-8}=2$, $U_{-7}=3, U_{-6}=4, U_{-5}=-0.6667, U_{-4}=-0.25, U_{-3}=1, U_{-2}=2, U_{-1}=3$, and $U_{0}=4$.

$$
\begin{aligned}
U_{12 n-3} & =\frac{U_{12 n-13} U_{12 n-9} U_{12 n-5}}{U_{12 n-11} U_{12 n-7}\left(-1-U_{12 n-13} U_{12 n-9} U_{12 n-5}\right)} \\
& =\frac{I A E}{(-A E I / C G(A E I+1)) C(-1-I A E)} \\
& =G, \\
U_{12 n-2} & =\frac{U_{12 n-12} U_{12 n-8} U_{12 n-4}}{U_{12 n-10} U_{12 n-6}\left(-1-U_{12 n-12} U_{12 n-8} U_{12 n-4}\right)} \\
& =\frac{J B F}{(-B F J / D H(B F J+1)) D(-1-J B F)} \\
& =H .
\end{aligned}
$$

Theorem 6. Every solution $\left\{U_{n}\right\}_{n=-9}^{\infty}$ of equation (28) is periodic with period six, and it is of the form

$$
\{A, B, C, D, E, F, A, \ldots\}
$$

iff

$$
\begin{gathered}
A=G, \\
B=H, \\
C=I, \\
D=J, \\
A E I=-2, \\
B F J=-2 .
\end{gathered}
$$




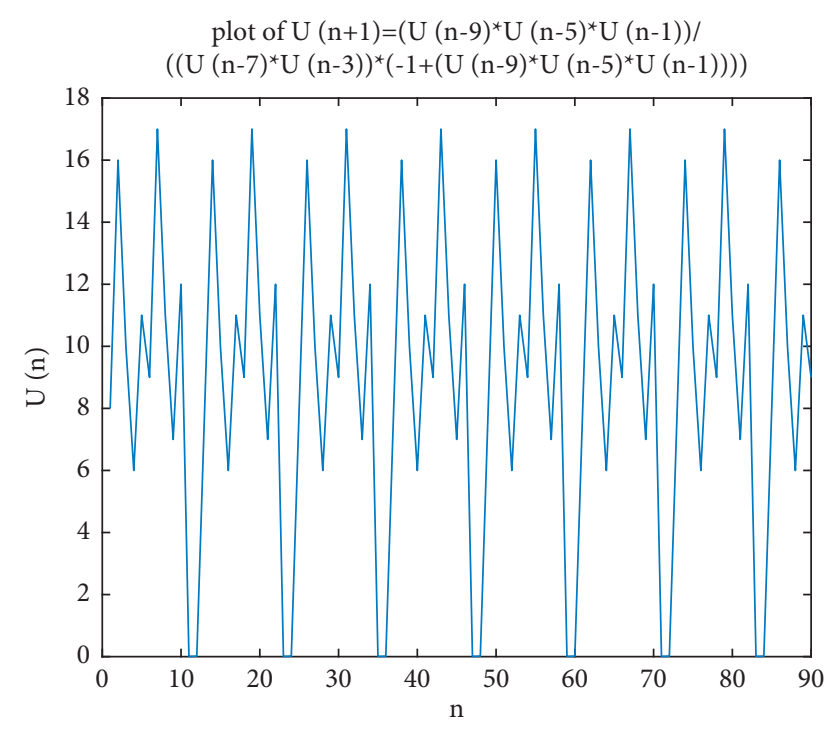

FIgURE 7: The solution of equation (35) with period twelve when $U_{-9}=8, U_{-8}=16, U_{-7}=10, U_{-6}=6, U_{-5}=11, U_{-4}=9, U_{-3}=17$, $U_{-2}=11, U_{-1}=7$, and $U_{0}=12$.

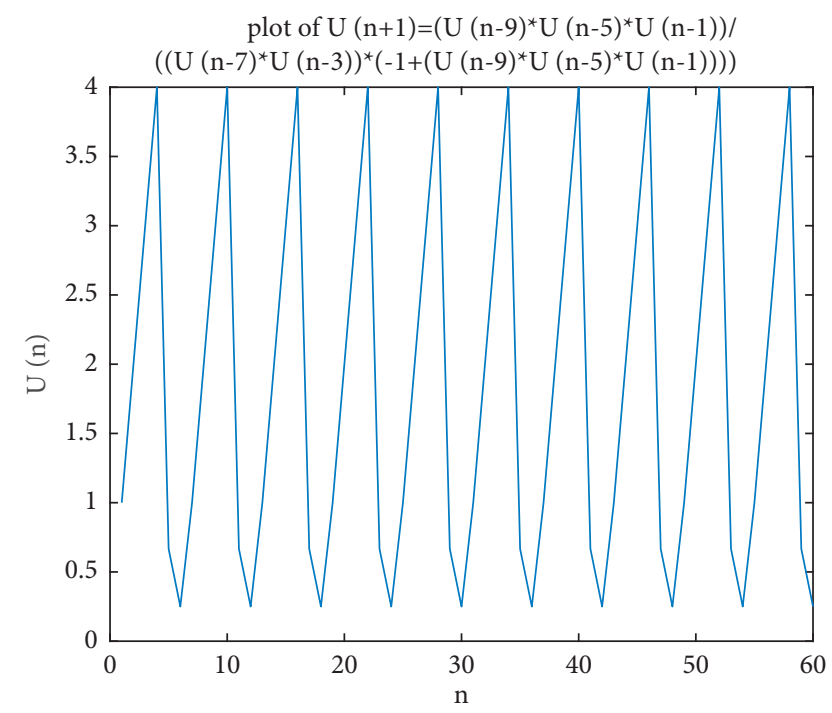

FIGURE 8: The solution of equation (35) with period six when initial values satisfies the conditions in Theorem 8, that are $U_{-9}=1$, $U_{-8}=2, U_{-7}=3, U_{-6}=4, U_{-5}=0.6667, U_{-4}=0.25, U_{-3}=1$, $U_{-2}=2, U_{-1}=3$, and $U_{0}=4$.

Example 5. We present numerical example for equation (28) for illustrating the results of this section where the initial conditions are $U_{-9}=8, U_{-8}=16, U_{-7}=10, U_{-6}=6$, $U_{-5}=11, U_{-4}=9, U_{-3}=17, U_{-2}=11, U_{-1}=7$, and $U_{0}=$ 12 (Figure 5).

Example 6. For confirming the results of this section, we consider numerical example for equation (28) where the initial conditions are $U_{-9}=1, U_{-8}=2, U_{-7}=3, U_{-6}=4$, $U_{-5}=-0.6667, U_{-4}=-0.25, U_{-3}=1, U_{-2}=2, U_{-1}=3$, and $U_{0}=4$ (Figure 6).

\section{Fourth Case:}

$$
\begin{aligned}
& U_{n+1}==U_{n-9} U_{n-5} U_{n-1} / U_{n-7} U_{n-3} \quad\left(-1+U_{n-9}\right. \\
& \left.U_{n-5} U_{n-1}\right)
\end{aligned}
$$

The solutions to difference equations

$$
U_{n+1}=\frac{U_{n-9} U_{n-5} U_{n-1}}{U_{n-7} U_{n-3}\left(-1+U_{n-9} U_{n-5} U_{n-1}\right)}, \quad n=0,1, \ldots,
$$

are studied in this section.

Theorem 7. Every solution $\left\{U_{n}\right\}_{n=-9}^{\infty}$ of equation (35) is periodic with period twelve, and it is in the form

$\left\{A, B, C, D, E, F, G, H, I, J,+\frac{A E I}{C G(A E I-1)},+\frac{B F J}{D H(B F J-1)}, A, B, \ldots\right\}$,

where $U_{-9}=A, U_{-8}=B, \quad U_{-7}=C, U_{-6}=D, U_{-5}=E$, $U_{-4}=F, U_{-3}=G, U_{-2}=H, U_{-1}=I$, and $U_{0}=J$.

Proof. The proof is identical to the method to prove Theorem 5.

Theorem 8. Every solution $\left\{U_{n}\right\}_{n=-9}^{\infty}$ of equation (35) is periodic with period six, and it is in the form

$$
\{A, B, C, D, E, F, A, \ldots\} .
$$

iff

$$
\begin{aligned}
A & =G, \\
B & =H, \\
C & =I, \\
D & =J, \\
A E I & =2, \\
B F J & =2 .
\end{aligned}
$$

We provide numerical examples of equation (35) for confirming our results.

Example 7. Assume that the starting conditions are as follows: $U_{-9}=8, U_{-8}=16, U_{-7}=10, U_{-6}=6, U_{-5}=11$, $U_{-4}=9, \quad U_{-3}=17, \quad U_{-2}=11, U_{-1}=7, \quad$ and $U_{0}=12$ (Figure 7).

Example 8. Let the initial conditions be given by $U_{-9}=1$, $U_{-8}=2, \quad U_{-7}=3, \quad U_{-6}=4, U_{-5}=0.6667, U_{-4}=0.25$, $U_{-3}=1, U_{-2}=2, U_{-1}=3$, and $U_{0}=4$ (Figure 8).

\section{Conclusion}

This research discussed the structure and behavior of solutions for four special cases of equation (1). In the second and third sections, we proved the stability of the equilibrium point. In the fourth and fifth sections, we obtained the periodic solutions to the equations with periodicity twelve. 
In addition, we studied the conditions of existence of the periodic solutions with period six.

\section{Data Availability}

The data used to support the findings of this study are included within the article.

\section{Conflicts of Interest}

The authors declare that they have no conflicts of interest.

\section{References}

[1] A. Khaliq, S. K. S. Hassan, M. Saqib, and D. S. Mashat, "Behavior of a seventh order rational difference equations," $D y$ namic Systems and Applications, vol. 28, no. 4, pp. 1056-2176, 2019.

[2] M. M. El-Dessoky, E. M. Elabbasy, and A. Asiri, "Dynamics and solutions of a fifth-order nonlinear difference equation," Discrete Dynamics in Nature and Society, vol. 2018, Article ID 9129354, 21 pages, 2018.

[3] M. Ghazel, E. M. Elsayed, A. E. Matouk, and A. M. Mousallam, "Investigating dynamical behaviors of the difference equation $x\{n+1\}=C x n-5 / A+B x n-2 x n-5$," The Journal of Nonlinear Science and Applications, vol. 10, no. 9, pp. 4662-4679, 2017.

[4] M. B. Almatrafi and M. M. Alzubaidi, "Qualitative analysis for two fractional difference equations," Nonlinear Engineering, vol. 9, no. 1, pp. 265-272, 2020.

[5] H. S. Alayachi, M. S. M. Noorani, A. Q. Khan, and M. B. Almatraf, "Analytic solutions and stability of sixth order difference equations," Mathematical Problems in Engineering, vol. 2020, Article ID 1230979, 12 pages, 2020.

[6] S. Sadiq, M. Kalim, and M. Kalim, "Dynamics of some higher order rational difference equations," International Journal of Advanced and Applied Sciences, vol. 5, no. 7, pp. 64-70, 2018.

[7] E. M. Elsayed and T. F. Ibrahim, "Solutions and periodicity of a rational recursive sequences of order five," Bulletin of the Malaysian Mathematical Sciences Society, vol. 38, no. 1, pp. 95-112, 2015.

[8] M. B. Almatrafi, "Solutions structures for some systems of fractional difference equations," Open Journal of Mathematical Analysis, vol. 3, no. 1, pp. 51-61, 2019.

[9] A. M. Alotaibi, M. S. M. Noorani, and M. A. El-Moneam, "On the asymptotic behavior of some nonlinear difference equations," Journal of Computational Analysis and Applications, vol. 26, pp. 604-627, 2019.

[10] E. Chatterjee, E. A. Grove, Y. Kostrov, and G. Ladas, "On the trichotomy character of," Journal of Difference Equations and Applications, vol. 9, no. 12, pp. 1113-1128, 2003.

[11] X. Yan and W. Li, "Global attractivity for a class of nonlinear difference equations," Soochow J. Math.vol. 29, no. 3, pp. 327-338, 2003.

[12] X. Yang, W. Su, B. Chen, G. M. Megson, and D. J. Evans, “On the recursive sequence," Applied Mathematics and Computation, vol. 162, no. 3, pp. 1485-1497, 2005.

[13] Y. Yazlik and M. Kara, "On a solvable system of difference equations of higher-order with period two coefficients," Communications Faculty of Science University of Ankara Series A1-Mathematics and Statistics, vol. 68, no. 2, pp. 1675-1693, 2019.
[14] L. Zhang, G. Zhang, and H. Liu, "Periodicity and attractivity for a rational recursive sequence," Journal of Applied Mathematics and Computing, vol. 19, no. 1-2, pp. 191-201, 2005. 